\title{
Breastfeeding knowledge among indigenous Temiar women: a qualitative study
}

\author{
Sharifah Zahhura Syed Abdullah ${ }^{1^{*}}$ \& Rozieyati Mohamed Saleh ${ }^{2}$ \\ ${ }^{1}$ Centre for Research on Women and Gender (KANITA), Universiti Sains Malaysia, \\ Pulau Pinang, Malaysia; ${ }^{2}$ Biomedical Science Programme, School of Health Sciences, \\ Universiti Sains Malaysia, Kelantan, Malaysia
}

\begin{abstract}
Introduction: The benefits of breastfeeding for both mothers and infants are widely recognised. Breastfeeding confirms a woman's unique ability to care for her infant in the best way possible and promotes optimum infant and maternal health. Methods: A qualitative research method involving five focus group discussions $(n=33)$ was chosen in this study to compare and contrast the breastfeeding practice in two different locations: the communities of Pos Pulat and the regroupment scheme settlement at Rancangan Pengumpulan Semula (RPS) Kuala Betis in Kelantan, Malaysia which represents different lifestyle experiences of indigenous Temiar population. Results: The benefits of breastfeeding to the infants reported by some Temiar women (42.4\%) were for the infant's health and growth. Responses from urban RPS Kuala Betis women include breast milk contains antibodies $(3.0 \%)$, delays in the return of regular ovulation (6.1\%), thus lengthening birth intervals and bonding between maternal-baby (6.1\%). In general, respondents from Pos Pulat seemed to have little knowledge regarding this issue, except for a woman who mentioned that maternal milk contains vitamins. Based on the narrative analysis, knowledge gap was observed between these two communities. Conclusion: Although all the women interviewed had the experience of breastfeeding their infants, most of them lacked the knowledge regarding the benefits of the breastfeeding either to the infants or to the mothers. The findings from this study are crucial for the preservation of breastfeeding culture among the Temiar women and can be used to improve promotion of breastfeeding to other Orang Asli groups in Malaysia.
\end{abstract}

Keywords: Breastfeeding, knowledge, health, indigenous, qualitative

\section{INTRODUCTION}

The Malay term Orang Asli that translates as "original people" or "first people" is the term given to indigenous people (Masron, Masami \& Ismail, 2013). Anthropologists, economists and planners describe the livelihoods of these indigenous peoples as "poor and marginalised minority groups"
(Habibah, Hamzah \& Mushrifah 2010) because they exist on the fringes of highly developed areas. The Orang Asli is generally classified under three main groups: Negrito, Senoi and Proto Malay, with each group comprising six subgroups with ethno-linguistic differences. The Temiar is one of the sub-ethnic groups of the Senoi people that can be found in the highest interior mountains

\footnotetext{
*Corresponding author: Dr Sharifah Zahhura Syed Abdullah

Centre for Research on Women and Gender (KANITA), Universiti Sains Malaysia,

Pulau Pinang, Malaysia

Tel: +6019-2322355; Fax: +604-6566379; E-mail: zahhura@usm.my

doi: https://doi.org/10.31246/mjn-2018-0103
} 
and at the headwaters of various rivers with limited access to civilisation (Domhoff, 2003). Nowadays, although most still live a traditional life, others have moved out of the forest to settle in more accessible locales. Some live in villages while others have migrated to towns and cities. They live by various combinations of swidden farming ('slash and burn' agriculture and crop rotation), hunting, gathering, fishing, and trading forest products. More recently, some men have started to undertake waged employment as day labourers. A few have successfully become lawyers, teachers, doctors or government employees.

The World Health Organization (WHO) recommends that all infants be exclusively breastfed from birth until six months of age and it be continued until two years of age or longer along with complementary foods (WHO, 2001). In the first six months of life breast milk should be an infant's sole and optimal source of nutrition as it is superior to all supplementations. The International Convention on the Rights of the Child 1990 endorsed by the United Nations, in Article 24, has recognised breastfeeding as one of the child's right (United Nation, 1990). Breastfeeding within the first one hour of life has been shown to reduce neonatal mortality by almost $22 \%$ (Edmond et al., 2006; Debes et al., 2013; NEOVITA Study Group, 2016).

A systematic review by Victora et al. (2016) indicates that there are no differences between rich and poor mothers in exclusive breastfeeding rates, in low and middle-income countries. They suggested that this was because rich mothers are adopting exclusive breastfeeding at a much faster rate than are poor mothers, although only 20 years ago, the poorer mothers had substantially higher rates of exclusive breastfeeding. Continued breastfeeding is still more common in poor than in wealthy mothers, but the rates seem to be dropping among the former while remaining stable in rich mothers. The protection of breastfeeding in the world's poorest populations is therefore a major priority. They also emphasised the importance of breastfeeding for all women and children, irrespective of their economic background.

As most mothers are able to breastfeed, they should be provided with accurate information and support from their families, communities, and healthcare providers. Currently, only limited data is available on the breastfeeding practices among the Orang Asli Senoi, and particularly for the Temiar sub-group. An earlier study conducted among 189 Semai women (other sub-ethnics of Senoi) found that the mean breastfeeding duration was up to 23 months (Khor, 1985). A study done a decade later found that although 95\% of Semai women had breastfed their infants, the average duration was found to be reduced to 17.9 months (Osman \& Zaleha, 1995). Similar study conducted on another Senoi sub-ethnic (the Mah Meri group) revealed that the mean period of breastfeeding was calculated to be around 11 months, although all the mothers in the study had started lactation within one hour of delivery (Wan Norlida et al., 2007).

Many studies have utilised quantitative methods such as knowledge, attitude and perception questionnaires to gain information on breastfeeding. However, in recent years, the use of qualitative methods such as interviews and focus group discussions has gained recognition in providing a better understanding of the feelings of individuals, their perceptions and misconceptions regarding breastfeeding information and issues (Tengku Alina et al., 2012). Furthermore, underlying beliefs are better captured through this type of qualitative studies (Rashidian, Eccles \& Russell, 2008). In view of 
this, this study aimed to investigate the knowledge among Temiar mothers regarding the breastfeeding practices in their cultural context.

\section{MATERIALS AND METHODS}

This was an exploratory investigation conducted in 2015, using qualitative methods via five focus group discussions. The two different locations chosen for this project represented the different lifestyle experiences and practices of the Temiar. The communities of Pos Pulat in Kelantan (a state that is located in the north-east of Peninsular Malaysia) represent the experiences of Temiar who live in the forest or close to it, approximately $2 \mathrm{~km}$ away. On the contrary, the communities in the Rancangan Pengumpulan Semula (RPS) regroupment settlement scheme, located in Kuala Betis in Gua Musang, Kelantan, represented those in transition from forest to urban living.

The participants in the project were women in the various households who were responsible for feeding the infants. An appropriate sample size for a qualitative study is one that adequately answers the research question. In practice, the number of required subjects usually becomes obvious as the study progresses, as new categories, themes or explanations stop emerging from the data (i.e. having achieved data saturation) (Marshall 1996).

In each location, participants were identified using a snowball approach. This sampling technique was chosen by taking into consideration the shyness and sensitivity of indigenous people. The Temiar were best approached by people that they already knew or familiar with rather than by outsiders or strangers. First, the local staff of the Department of Orang Asli Development (JAKOA) and Tok Batin were contacted. The researcher explained the research project to the contact person and asked to identify potential participants. Each contact person was a local person who was active within or had connections to the Temiar community in that location. They knew of the best means to approach the target population and of any possible obstacles that might occur. The inclusion criteria were women of any age group who had breastfed a child in each location. Those who willingly consented to the study were enrolled and the times for focus group discussions were arranged.

Focus group discussion was selected as a suitable method for this project in order to gain in-depth understanding on a specific topic of interest (Nyumba et al., 2018). In this instance, focus group discussions helped to reveal the benefits of breastfeeding through active and live discussion between the participants and a trained facilitator. An "ice-breaker" or introduction was conducted before commencing each discussion session to create a friendly environment. Firstly, the facilitator introduced herself and broadly outlined the aims of the research and the protocols. The participants then introduced themselves and were asked to respond to the first question from the focus group schedule. According to Peterson \& Barron (2007), encouraging participants to become involved in an interactive, open discussion is one of the major challenges for the focus group facilitator.

Focus group discussions in Pos Pulat took place on a veranda of the home of one of the participants, while in RPS Kuala Betis it was conducted in the childcare centre (Taska) during weekend. All focus group discussions took place in a familiar comfortable environments in order to motivate participants, with the hope that the best possible responses could be obtained.

The questions for focus groups were developed to ensure consistency in responses according to project themes while allowing for flexibility of later 
interpretations. They were also designed to be culturally compatible and general rather than personally probing in nature. Research was conducted in ways that were appropriate for Orang Asli Temiar. It is important that participants felt at ease and not threatened by the researcher or the data-collection instruments. The focus group discussions started with a general discussion about the demographic profiling, followed by a discussion on benefits of breastfeeding to both baby and mother, their decision to breastfeed the baby and the women's expectation regarding breastfeeding practices.

There were five focus group discussions, of which two groups were conducted in Pos Pulat $(n=12)$ and three $(n=21)$ groups took place in RPS Kuala Betis. Each focus group discussions took about 45 minutes to one hour to complete. All participants were given false names for anonymity. Narrative analysis was done after every recording. All focus group discussions were audio-taped and video recorded (with permission) to allow the facilitator to focus on the group responses and non-verbal behaviour and these were then transcribed from oral recordings to written text. The aim was for participants to ignore the recording so the interaction would be natural. All the transcripts were coded and categorised as either a main or sub category and then 'thematised' using the software package for handling qualitative data, NVivo 10. The protocol of this study has been approved by the Human Research Ethics Committee of the Universiti Sains Malaysia (approval code FWA Reg. No.: 00007718; IRB Reg. No.: IRB00004494).

\section{RESULTS}

Four key themes emerged from the analyses of this study. These were the perception of benefits of breastfeeding to the infants and to the mothers, the decision of mothers to breastfeed their babies and the expectation of Temiar women pertaining to breastfeeding issue. Below are summaries of responses from women in both locations for each of these themes. Where relevant, some excerpts are provided to illustrate the responses and reflect the way of thinking of these women.

\section{Perception of the benefits of breastfeeding to the infants}

The perceived benefits of breastfeeding to the infants reported by some of the Temiar women in Pos Pulat and RPS Kuala Betis included the protection against a variety of infections as well as for infant health and growth. Below are some excerpts of the responses from women in both locations:

"For example the infection of disease was lesser... sometimes if the baby suck on breastmilk (sic), the occurrence of diarrhoea is less..." (Along, 36 years old, RPS Kuala Betis).

"Healthy... for baby's health" (Saadiah, 32 years old, RPS Kuala Betis).

"If we give milk such as formula milk there will be problem of diarrhoea... but if we give breastmilk, if we really do that, the baby will be fine" (Halimah, 45 years old, RPS Kuala Betis).

"For the baby's health... he will grow healthily. He will become clever" (Anum, 25 years old, Pos Pulat).

One woman from RPS Kuala Betis even mentioned that breast milk contains antibodies to fight diseases. Another woman from Pos Pulat also stated that mother's milk was the best food for the baby as it contained vitamins. She further added that she obtained the information from a pamphlet found at the hospital.

The practice of discarding colostrum was also discussed. The women from both locations were also asked whether 
they provide the first milk or colostrum to the baby. Most women from RPS Kuala Betis mentioned that they had given colostrum to their babies. In contrast, mothers from Pos Pulat agreed that colostrum needed to be discarded and thus revealed their limited knowledge about its nutritious and anti-infective properties. They believed that it was dirty and contained bacteria. Nevertheless, some of the women from Pos Pulat did mention that they will discard the colostrum when they gave birth at home, but gave it to the baby if the delivery was at a hospital.

\section{Perception of the benefits of breastfeeding to the mothers}

The women in the twolocations responded differently when asked regarding benefits of breastfeeding to mothers. The women in RPS Kuala Betis spoke of the various advantages. One woman mentioned the delay in the return of regular ovulation, thus lengthening birth intervals as one of the benefits. Another woman said that "it was much easier to breastfeed the baby compared to bottle feeding since it was not necessary to boil the water, because cleanliness was guaranteed with mother's milk". This view was also supported by another woman who stated that "moreover, we think why we need to pay for something that is already there? No need to buy bottle (formula) milk, no need to think. God has given everything complete in our body".

On the contrary, most of the women in Pos Pulat had no idea when asked about the benefits of breastfeeding to mothers. Only one woman responded that breastfeeding is to strengthen the bond between the mother and baby.

\section{Decision to breastfeed}

When the women were asked regarding their decision to breastfeed the baby, all of the women from both locations stated that they choose to breastfeed their babies when they realised that they were pregnant. Kinah from Pos Pulat said that "for example myself... aahhh... for me if someone... for example myself... if I am pregnant, when I deliver the baby... of course I will give breast milk... my own milk.... and no formula...".

A woman from RPS Kuala Betis said "I feel that when breast milk is given, the affection between us and the baby is more". Another woman added "this is how our mother did for us... their sacrifices... how a mother scarifies... now we feel it'. This was supported by another woman from RPS Kuala Betis who mentioned that she herself decided to give breast milk "when the baby was born, immediately start to breastfeed, it is compulsory, to give breast milk".

\section{Expectations regarding breastfeeding}

The majority of the mothers in Pos Pulat revealed that information about breastfeeding and infant formula was rarely provided by their obstetricians during prenatal visits. One respondent expressed the hope that health practitioners would give them some information regarding breastfeeding besides conducting vaccinations or routine check-ups:

"If it is okay we want the health people to come to give talk, talk regarding breastfeeding... because we only know how to give breast milk but we have no idea what the milk is good for... we know it is to cease hunger, to make the baby feels full and to make them healthy... and that's all... other than that we do not know..." (Kinah, 33 years old, Pos Pulat).

\section{DISCUSSION}

It has been well documented that the general health of the indigenous people is disproportionately lower than that of other communities. Although Orang Asli in Malaysia comprise only $0.6 \%$ of the 
total Malaysian population (Tan, 2016), they lag behind in almost all socioeconomic indicators (Nicholas, 2007). Numerous studies in Malaysia have reported these health inequalities. The Orang Asli, inter alia, have high infant mortality rates and lower life expectancy compared to the national standards. Compared with other communities, they are more malnourished and have a higher prevalence of persistent infectious diseases such as malaria, tuberculosis and skin diseases (Nicholas, 2007; Khor \& Zalilah, 2008).

Breastfeeding is a natural practice that has been shown to have numerous benefits not only to babies, but also to the mothers, society, economy and environment (Binns, Lee \& Low, 2016; U.S. Department of Health and Human Services, 2011). In this study, the Temiar women in RPS Kuala Betis, a semi-urban setting, seemed to have more knowledge regarding the benefits of breastfeeding compared to the mothers from the more rural setting of Pos Pulat due to their amenities and location. There was one Mothers and Child Clinic that can be found in RPS Kuala Betis, while there was none in Pos Pulat. The nurses in this clinic had a routine weekly or monthly talk on various topics that involved mothers and child health issues. Thus women in RPS Kuala Betis had better opportunities to gain more information and knowledge compared to their counterparts in Pos Pulat. In contrast to this, the health practitioners visited Pos Pulat only once a month and only performed routine check-ups. According to the participants of this study, there were no talks or information given on breastfeeding and its benefits or on any other general health topics. In this particular case at least, the healthcare provider had overlooked the need for disseminating such basic but vital information to this community. The lack of physical support such as this had a negative impact. Since it is far-fetched to expect this rural community to gain online information, they are dependent on support programmes such as health talks, discussions, pamphlets and hardcopy materials. Indigenous people are known to have low self-esteem and are shy and timid (Louth, 2012). As such, it is very unlikely that they will initiate any discussion and enquiries, despite their curiosity and eagerness to learn. Gaps in breastfeeding knowledge will thus remain unfilled among them.

Studies have suggested that mothers who are knowledgeable about the health benefits of breastfeeding are more likely to breastfeed (DiGirolamo et al., 2005; McCann, Baydar \& Williams, 2007). Research has shown that mothers still believed that breastfeeding is the best practice for their babies, despite knowing less about the specific reductions in health risks that occur through breastfeeding and the consumption of breast milk (McCann et al., 2007). This study found that despite having limited or almost no knowledge of the benefits of breastfeeding, most women, particularly those in Pos Pulat still nursed their babies. For them, breastfeeding was a cultural norm which had been entrenched in their society. It also revealed that the Orang Asli women still held on firmly to their values and trusted the knowledge that was passed on to them from family members and women within their community, which included the practice of breastfeeding. In addition, the desire to give the best for their babies was the key driving force for these women throughout their decision-making process. Even though breastfeeding is often described as natural, it is actually an art that has to be learned by both the mother and the newborn. Skills on how to hold and position a baby at the breast, how to achieve an effective latch, and other breastfeeding techniques may need to 
be taught (U.S. Department of Health and Human Services, 2011) in order to achieve effective lactation. Women and their families need to be informed fully on the health benefits of breastfeeding for infants and their mothers (Sridhar \& Salcedo, 2017). Furthermore, it is essential to impart additional knowledge about the need for patience, the difficulties associated with beginning of breastfeeding and the acceptance of these difficulties as a normal part of the process (Giugliani, 2004). This will ensure that the practice is sustainable for optimal periods. Maternal knowledge, understanding, confidence, self-efficacy and being proactive in seeking support and education have a positive correlation with the longer duration and exclusivity of breastfeeding (Brown, 2014).

It is also known that breastfeeding provides a sense of bonding, wellbeing, and improved self-esteem for many women (Labbok, 2001). Breastfeeding too is associated with increased child spacing in women who practised breastfeeding compared to those who did not. Fertility is not immediately recovered with the return of regular menstrual cycles. The length of postpartum amenorrhea varies greatly and depends on several factors, including maternal age and parity as well as the duration and frequency of breastfeeding (Chao, 1987). However this alone cannot provide a means of reliable and adequate contraception for most women.

Breast milk is the perfect source of nutrition for infants, both in its nutritional composition and in the non-nutritive bioactive factors that are needed for infant survival and healthy development (Ballard \& Morrow, 2013). The first fluid produced by mothers after delivery (colostrum), is rich in immunologic components such as secretory of immunoglobulin A (IgA), lactoferrin, leucocytes, as well as developmental factors such as epidermal growth factor (Castellote et al., 2011). There are various cultural practices with regard to discarding or feeding colostrum to the newborn. In our study, all women in Pos Pulat agreed that they needed to discard the colostrum because they believed that it is dirty and contaminated with bacteria. Other studies have also reported on the avoidance of giving this substance to the infants due to the cultural beliefs and taboos. A study in Chandigarh found that majority of the mothers considered colostrum as 'dirty, yellow, smelly, stagnant milk' which should not be given to the infant because of its impurity as it is produced during the antenatal period (Walia, Kalia \& Chopra, 2009). The reasons for discarding colostrum vary with different cultures, but most societies believe that colostrum is dirty, poisonous and contaminated (Liamputtong, 2007). Domestic data have shown that more than $60 \%$ of Mah Meri women discarded their colostrum because of tradition, the perception that it was filthy and could cause illness to the baby, discomfort and fullness of the breasts, advice by others or for no reason at all. Some respondents also claimed that besides being told by close families and friends to discard the colostrum, they also received similar advice from some of the professional health workers (doctors and nurses) (Wan Norlida et al., 2007).

In the present study, the women in Pos Pulat who delivered at the hospital gave colostrum to their babies following encouragement from the hospital staff and the information that they had gained from reading the available pamphlets. Knowledge pertaining to benefits of giving first milk to the babies for women in RPS Kuala Betis had been communicated to them by nurses at the clinic. Given that the knowledge and benefits of breastfeeding are sub-optimal among the rural Orang Asli, there is a pressing need for the health authorities 
to play an active role in promoting the practice to help bridge the gap in the health and social disparities between rural and urban communities. Moreover it is also imperative that healthcare workers are equipped with accurate knowledge and appropriate standards, and provide equitable delivery of it, so that conflicting advice can be avoided.

In our study, all women from both locations were confident with their decision to breastfeed. Confidence in breastfeeding is an indication that she possesses the knowledge and skills to successfully breastfeed her infant. It is based on information gained from prior experience, the observations of other women who had breastfed, support and encouragement from individuals whose opinions are respected, and the physiological reaction to the prospect or act of breastfeeding (Dennis \& Faux, 1999). Since confidence is enhanced through knowledge, breastfeeding education, particularly when it is skills-based or observational, may be an important strategy for improving breastfeeding rates (Chezem, Friesen \& Boettcher, 2003). Although the women in this study were confident enough to breastfeed their babies, the lack of knowledge regarding the benefits of the breastfeeding may result in decreasing rates of it in the future. Maternal awareness can be compromised if important information is not received. Moreover, many people, including some health professionals, believe that because commercially prepared formula has improved in recent years, this form of sustenance is equivalent to breast milk in terms of its quality and health benefits (U.S. Department of Health and Human Services, 2011; Martin, Ling \& Blackburn, 2016).

This can be quite alarming since this disadvantaged group would never question the actions of health professionals, they can easily be influenced and accept any advice from promoters of formula-feeding (MacVicar et al., 2015). Furthermore, it was also noted that mothers from low socioeconomic and education background may require substantial input and support surrounding the breastfeeding issues. As such, it is highly critical for health practitioners to possess good interpersonal communication skills, as well as to master some basic fundamental knowledge on breastfeeding, while tackling some misconception of this practice in cultural context in order to ensure optimal breastfeeding practice. Inaccurate and contradictory information can easily cause early cessation of this practice in this community (MacGregor \& Hughes, 2010).

The WHO International Code of Marketing Breastmilk Substitutes was adopted in 1981 by the World Health Assembly (WHA) to contribute to the provision of safe and adequate nutrition for infants, by the protection and promotion of breastfeeding, and by ensuring the proper use of breast-milk substitutes, when these are necessary (WHO, 1981). In order to protect, support and promote breastfeeding practices in Malaysia, Ministry of Health (MOH) first formulated a Code of Ethics for Infant Formula Products in 1979 based on 1975 International Council of Infant Food Industries (ICIFI) Code. A revised version of the Code was launched on August 1, 2008 and was renamed Code of Ethics for the Marketing of Infant Foods and Related Products $(\mathrm{MOH}$, 2019). The goal of the Code of Ethics' is to contribute to the promotion of safe and optimal nutrition for infants by protecting and promoting breastfeeding and by ensuring the use of correct breast milk replacement products where this is necessary, by providing adequate 
information using a controlled marketing and distribution method.

Although Malaysia has some of the strictest regulations that protect the practice of breastfeeding, some companies which market formula milk continue to circumvent the Code. This has resulted in numerous violations of the International Code of Marketing of Breast-milk Substitutes and subsequent resolutions by IBFAN (2014) that have been documented over the years. A compilation of violations includes direct promotion and selling of their formula milk products to parents at Parenthood Expos. Other violations are more subtle. These include attracting parents with lucrative packages with every purchase of their formula milk products, encouraging parents to submit videos of their kids with product logos and reviews. The promotion of formula milk with includes comprehensive programmes that cover the entire period of pregnancy to the toddler stages with the recommended company's products in every developmental stage, and promotion in a form of goodie bags, coupons and prizes. Events related to bottle-feeding are heavily promoted through social media such as Facebook. In clinics in Malaysia, particularly at private practices, formula names and brand logos are promoted in the form of decorative accessories, toys and formula milk tester size.

Besides skilful marketing strategies to promote formula-milk feeding, some bottle-feeding accessories such as teats are sold without any indication that its use will interfere with the breastfeeding practices as required by the Malaysian Code. Such items are often wrapped in attractive packaging with claims and comforting words that the products are "ideal for babies", are "anti-colic teats", "natural breast-shaped teats", "natural latch", etc.
The old tradition of breastfeeding among Temiar women can easily be eroded if marketing that favours bottlefeeding reaches them. This, together with their limited knowledge of the benefits of breast milk can result in a decrease or even cessation of their breastfeeding practices. This would be unfortunate since Orang Asli groups have more to gain from extended breastfeeding and where breast milk is the most secure and economically advantageous way to feed infants.

\section{CONCLUSION}

This study analyses the descriptions of past events and provides insights into the thoughts, emotions, interpretations and lived experiences of the Temiar women regarding breastfeeding. Our study has revealed that there is a knowledge gap between rural and semi-urban Temiar women despite originating from the same sub-ethnic background. Mothers from semi-urban setting of RPS Kuala Betis seem to have better awareness of the vast array of the benefits of breastfeeding compared to those from the rural Pos Pulat location. Besides these, inequalities in the healthcare services provided to both locations were also noted in this study. There was inadequate support for those in Pos Pulat, where dissemination of breastfeeding knowledge or on any other health topics was neglected by the healthcare provider. Fortunately, due to the strong cultural norms within this community, the practice of breastfeeding has continued despite having limited awareness of the benefits of such a tradition. It is vital to inculcate strong fundamental knowledge and reinforce beliefs of benefits of breastfeeding practice among the Temiar for preservation of their breastfeeding culture, in the face of subtle and direct pressures for bottle/ formula-feeding from outside. Policy makers and healthcare providers should 
ensure that accurate information on breastfeeding is disseminated and made accessible to Orang Asli women through qualified health practitioners in order to make breastfeeding practice sustainable.

This study had a limited number of participants and was carried out only among the Temiar, a sub-ethnic group of the Orang Asli. Therefore it is suggested that more research should be carried out to explore the breastfeeding practices among other 17 sub-ethnic groups of Orang Asli in Peninsular Malaysia. The findings from this study can be used to improve promotion of breastfeeding to mothers of other Orang Asli groups in Malaysia.

\section{Acknowledgement}

This study was made possible by the generous support of the Universiti Sains Malaysia Short Term Grant (304/PKANITA/61313051). We would also like to thank the Department of Orang Asli Development (JAKOA) Malaysia for their assistance in this study.

\section{Authors' contributions}

SZSA, principal investigator who conceptualized and designed the study, led the data collection, data analysis and interpretation, prepared the draft of the manuscript and reviewed the manuscript; RMS, assisted in the drafting of the manuscript, review of the data analysis and interpretation and also reviewed the final manuscript.

\section{Conflict of interest}

The authors declare that there was no conflict of interest in the publication of this paper.

\section{References}

Ballard O \& Morrow AL (2013). Human milk composition: nutrients and bioactive factors. Pediatr Clin N Am 60(1):49-74.

Binns C, Lee M \& Low WY (2016). The Long-Term Public Health Benefits of Breastfeeding. Asia Pac J Public Health 28(1):7-14.

Brown A (2014). Maternal trait personality and breastfeeding duration: the importance of confidence and social support. $J$ Adv Nurs 70(3):587-98.
Castellote C, Casillas R, Ramírez-Santana C, PérezCano FJ, Castell M, Moretones MG, LópezSabater MC \& Franch A (2011). Premature delivery influences the immunological composition of colostrum and transitional and mature human milk. J Nutr 141(6):1181-7.

Chao S (1987). The effect of lactation on ovulation and fertility. Clin Perinatol 14(1):39-50.

Chezem J, Friesen C \& Boettcher J (2003). Breastfeeding knowledge, breastfeeding confidence, and infant feeding plans: effects on actual feeding practices. J Obstet Gynecol Neonatal Nurs 32(1):40-7.

Debes AK, Kohli A, Walker N, Edmond K \& Mullany LC (2013). Time to initiation of breastfeeding and neonatal mortality and morbidity: a systematic review. BMC Public Health 13(Suppl 3):S19.

Dennis CL \& Faux S (1999). Development and psychometric testing of the Breastfeeding SelfEfficacy Scale. Res Nurs Health 22(5):399-409.

DiGirolamo A, Thompson N, Martorell R, Fein S \& Grummer-Strawn L (2005). Intention or experience? Predictors of continued breastfeeding. Health Educ Behav 32(2):20826.

Domhoff GW (2003). In: Senoi dream theory: myth, scientific method, and the dreamwork movement. From http://dreamresearch.net/ Library/senoi.html. [Retrieved November 18 2017].

Edmond KM, Zandoh C, Quigley MA, AmengaEtego S, Owusu-Agyei S \& Kirkwood BR (2006). Delayed breastfeeding initiation increases risk of neonatal mortality. Pediatrics 117(3):e380-6.

Giugliani ER (2004). Common problems during lactation and their management. $J$ Pediatr (Rio J) 80(Suppl 5):S147-54.

Habibah A, Hamzah J \& Mushrifah I (2010). Sustainable livelihood of the community in Tasik Chini biosphere reserve: the local practices. Journal of Sustainable Development 3(3):184-196.

IBFAN (2014). BTR in brief. Evidence of Violations of the International Code of Marketing of Breastmilk Substitutes and subsequent resolutions compiled from January 2011 to December 2013. IBFAN Sdn. Bhd.

Khor GL (1985). A Study of the Nutritional Status of the Semai. Ph.D. Thesis, Universiti Malaya, Kuala Lumpur. 
Khor GL \& Zalilah MS (2008). The Ecology of Health and Nutrition of "Orang Asli" (Indigenous People) Women and Children in Peninsular Malaysia. Tribes and Tribals Special Volume(2):67-77.

Labbok MH (2001). Effects of breastfeeding on the mother. Pediatr Clin N Am 48(1):143-158.

Liamputtong $P$ (2007). Childrearing and infant care issues: a cross-cultural perspective. Nova Science Publishers, New York.

Louth S (2012). Overcoming the 'shame' factor: empowering indigenous people to share and celebrate their culture. In International Conference: Innovative Research in a Changing and Challenging World: Conference Proceedings (B). Australian Multicultural Interaction Institute. From https://eprints. usq.edu.au/23010/. [Retrieved November 18 2017].

MacGregor E \& Hughes M (2010). Breastfeeding experiences of mothers from disadvantaged groups: a review. Community Pract 83(7):30-3.

MacVicar S, Kirkpatrick P, Humphrey T \& ForbesMcKay KE (2015). Supporting breastfeeding establishment among socially disadvantaged women: a meta-synthesis. Birth 42(4):290-8.

Marshall MN (1996). Sampling for qualitative research. Family Practice 13:522-525.

Martin CR, Ling PR \& Blackburn GL (2016). Review of infant feeding: key features of breast milk and infant formula. Nutrients 8(5):279.

Masron T, Masami F \& Ismail N (2013). Orang Asli in Peninsular Malaysia: population, spatial distribution and socio-economic condition. $J$ Ritsumeikan Soc Sci Humanit 6:75-115.

McCann MF, Baydar N \& Williams RL (2007). Breastfeeding attitudes and reported problems in a national sample of WIC participants. $J$ Hum Lact 23(4):314-24.

MOH (2019). Code of Ethics in Marketing Infant Food and Related Products. Ministry of Health Malaysia. From http://nutrition.moh.gov.my/ en/tata-etika-pemasaran-makanan-bayi-danproduk-berkaitan/. [Retrieved January 30 2019].

NEOVITA Study Group (2016). Timing of initiation, patterns of breastfeeding, and infant survival: prospective analysis of pooled data from three randomised trials. Lancet Glob Hlth 4(4):e266-75.
Nicholas C (2007). The Orang Asli and the Contest for Resources. Indigenous Politics, Development and Identity in Peninsular Malaysia. Center for Orang Asli Concerns \& IWGIA, Kuala Lumpur.

Nyumba TO, Wilson K, Derrick CJ \& Mukherjee N (2018). The use of focus group discussion methodology: insights from two decades of application in conservation. Methods Ecol Evol 9:20-32.

Osman A \& Zaleha MI (1995). Nutritional status of women and children in Malaysian rural populations. Asia Pac J Clin Nutr 4(3):319-24.

Peterson ER \& Barron KA (2007). How to get focus groups talking: new ideas that will stick, International Journal of Qualitative Methods 6(3):140-144.

Rashidian A, Eccles MP \& Russell I (2008). Falling on stony ground? A qualitative study of implementation of clinical guidelines' prescribing recommendations in primary care. Health Policy 85:148-161.

Sridhar A \& Salcedo J (2017). Optimizing maternal and neonatal outcomes with postpartum contraception: impact on breastfeeding and birth spacing. Matern Health Neonatol Perinatol 3:1.

Tan V (2016). In: Getting to know indigenous Malaysians. From https://www.thestar.com. my/metro/community/2016/08/09/gettingto-know-indigenous-msians-facts-at-a-glanceglimpse-into-the-lives-of-selangors-two-mainora/. [Retrieved November 20 2017].

Tengku Alina TI, Zaharah S, Rohana J, Wan Manan WM \& Nik Normanieza NM (2012). Breast milk expression among formally employed women in urban and rural Malaysia: A qualitative study. International Breastfeeding Journal 7:11-21.

United Nation (1990). In: Convention on the Rights of the Child. From https://www.ohchr.org/en/ professionalinterest/pages/crc.aspx [Retrieved January 10 2018].

U.S. Department of Health and Human Services (2011). In: The Surgeon General's Call to Action to Support Breastfeeding. Washington, DC: U.S. Department of Health and Human Services, Office of the Surgeon General. From https:// www.ncbi.nlm.nih.gov/books/NBK52682/. [Retrieved January 18 2018]. 
Victora CG, Bahl R, Barros AJD, França GVA, Horton S, Krasevec J, Murch S, Sankar MJ, Walker N \& Rollins NC (2016). Breastfeeding in the 21st century: epidemiology, mechanisms, and lifelong effect. Lancet 387: 475-90.

Walia I, Kalia R \& Chopra S (2009). Initiation of breast feeding - The cultural factors. Nursing and Midwifery Research Journal 5(1):10-18.

Wan Norlida, WN, Zalilah MS, Khor, GL, Ng, WC, Mirnalini, K, Nawalyah, AG, \& Hejar, AR (2007). Breastfeeding practices and nutritional status of Orang Asli children (Temuan and Mah Meri) in Sepang District and Carey Island, Selangor. Mal J of Med and Health Sci, 3(2):1-15.
WHO (1981). International Code of Marketing of Breast-milk Substitutes. WHO, Geneva.

WHO (2001). In: The World Health Organization's infant feeding recommendation. From http:// www.who.int/nutrition/topics/infantfeeding recommendation/en/index.html. [Retrieved October 10 2017]. 